\title{
OPEN Association between pulse pressure and progression of chronic kidney disease
}

\author{
Toshiki Maeda ${ }^{1 凶}$, Soichiro Yokota ${ }^{2}$, Takumi Nishi ${ }^{3}$, Shunsuke Funakoshi ${ }^{1}$, Masayoshi Tsuji ${ }^{4}$, \\ Atsushi Satoh ${ }^{1}$, Makiko Abe ${ }^{1}$, Miki Kawazoe ${ }^{1}$, Chikara Yoshimuraa ${ }^{1}$, Kazuhiro Tada ${ }^{2}$, \\ Koji Takahashi ${ }^{2}$, Kenji Ito ${ }^{2}$, Tetsuhiko Yasuno ${ }^{2}$, Toshitaka Yamanokuchi ${ }^{5}$, Kazuyo Iwanaga ${ }^{1}$, \\ Akiko Morinaga ${ }^{1}$, Kaori Maki ${ }^{1}$, Tamami Ueno ${ }^{1}$, Kousuke Masutani ${ }^{2}$, Shigeaki Mukoubara ${ }^{6}$ \& \\ Hisatomi Arima $^{1}$
}

The aim of this study was to investigate the association between pulse pressure (PP) and chronic kidney disease (CKD) progression among the general population in Japan. We conducted a populationbased cohort study of the residents of Iki Island, Nagasaki, Japan, from 2008 to 2018. We identified 1042 participants who had CKD (estimated glomerular filtration rate(eGFR) $<60 \mathrm{~mL} / \mathrm{min} / 1.73 \mathrm{~m}^{2}$ or the presence of proteinuria) at baseline. Cox's proportional hazard model was used to evaluate the association between PP and progression of CKD. During a 4.66-year mean follow-up, there were 241 cases of CKD progression (incident rate: 49.8 per 1000 person-years). A significant increase existed in CKD progression per $10 \mathrm{mmHg}$ of PP elevation, even when adjusted for confounding factors [adjusted hazard ratio $1.17(1.06-1.29) p<0.001$ ]. Similar results were obtained even after dividing PP into quartiles [Q2: $1.14(0.74-1.76), 03: 1.35(0.88-2.06), 04: 1.87(1.23-2.83) p=0.003$ for trend]. This trend did not change significantly irrespective of baseline systolic or diastolic blood pressures. PP remained a potential predictive marker, especially for eGFR decline. In conclusion, we found a significant association between PP and CKD progression. PP might be a potential predictive marker for CKD progression.

Chronic kidney disease (CKD) is characterized by renal function decline and/or the presence of albuminuria ${ }^{1}$. $\mathrm{CKD}$ is an important and established risk factor for end-stage renal disease (ESRD) and renal replacement therapy ${ }^{2,3}$. CKD also leads to cardiovascular disease or mortality ${ }^{4,5}$. Patients with CKD incur tremendous health care costs ${ }^{6,7}$; thus, CKD is a highly prioritized public health issue.

Some conditions such as high blood pressure ${ }^{8-10}$, hyperglycemia ${ }^{11-13}$, and smoking ${ }^{14,15}$ are thought to be risk factors for CKD progression. Among them, high blood pressure is one of the most established prognostic factors for CKD progression ${ }^{6-8}$. Pulse pressure (PP), which is easily calculated by subtracting the diastolic blood pressure (DBP) from systolic blood pressure (SBP), is a potential marker for atherosclerotic diseases including cardiovascular disease ${ }^{16-20}$, heart failure ${ }^{16,21}$, and stroke ${ }^{16}$. It was suggested that PP might be a superior predictor for coronary heart disease compared with SBP and $\mathrm{DBP}^{20}$. Thus, PP might also detect CKD progression effectively. However, few studies have investigated $\mathrm{PP}$ as a risk factor for CKD progression, especially in a general population.

Thus, the aim of this study was to investigate the association between PP and CKD progression among the general population in Japan.

\footnotetext{
${ }^{1}$ Department of Preventive Medicine and Public Health, Faculty of Medicine, Fukuoka University, 7-45-1 Nanakuma, Jonan-ku, Fukuoka 814-0180, Japan. ${ }^{2}$ Department of Internal Medicine, Division of Nephrology and Rheumatology, Faculty of Medicine, Fukuoka University, Fukuoka, Japan. ${ }^{3}$ Department of Research Planning and Information Management, Fukuoka Institute of Health and Environmental Sciences, Fukuoka, Japan. ${ }^{4}$ Department of Lifestyle and Welfare Information, Kindai University Kyushu Junior College, Fukuoka, Japan. ${ }^{5}$ Department of Physical Therapy, Faculty of Medical Science, Fukuoka International University of Health and Welfare, Fukuoka, Japan. ${ }^{6}$ Department of Internal Medicine, Nagasaki Prefecture Iki Hospital, Nagasaki, Japan. ${ }^{\bowtie}$ email: tmaeda@ fukuoka-u.ac.jp
} 


\begin{tabular}{|c|c|c|c|c|}
\hline & & \multicolumn{3}{|c|}{ Proteinuria categories } \\
\hline & & $\mathrm{A} 1$ & $\mathrm{~A} 2$ & A3 \\
\hline GFR categories & $\begin{array}{c}\text { eGFR } \\
\left(\mathrm{m} / \mathrm{min} / 1.73 \mathrm{~m}^{2}\right)\end{array}$ & $(-) /( \pm)$ & $(1+)$ & $(2+)-$ \\
\hline G1 & $\geq 90$ & & & 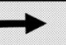 \\
\hline G2 & $60-89$ & & & \\
\hline G3a & $45-59$ & I & & \\
\hline G3b & $30-44$ & & & \\
\hline G4 & $15-29$ & & & \\
\hline G5 & $<15$ & & & \\
\hline
\end{tabular}

Figure 1. Definition of outcomes. The colored area was eligible for study (those with CKD at baseline). Progression of CKD was defined as exacerbation in the eGFR category or urinary protein category from baseline categories according to the Kidney Disease: Improving Global Outcome (KDIGO) CKD guidelines.

\begin{abstract}
Methods
Study design and participants. The Iki epidemiological Study of atherosclerosis and chronic kidney disease (ISSA-CKD) is a population-based cohort study of the residents of Iki Island, Nagasaki Prefecture, Japan. The details of the project have been described elsewhere ${ }^{22}$. Briefly, this project was started in 2016 and designed to decrease ESRD in Iki Island, where the rate of those undergoing hemodialysis was previously the highest in Nagasaki Prefecture. We conducted an observational study using health check-up data to help prevent CKD and atherosclerosis disease in Iki Island. From 2008 to 2018, 8029 residents underwent annual health check-ups conducted in Iki City. After the exclusion of 1856 residents who attended only once, 253 who had missing serum creatinine values or urine findings, and 4878 who did not have CKD at baseline, 1042 participants who had CKD (estimated glomerular filtration rate $(\mathrm{eGFR})<60 \mathrm{~mL} / \mathrm{min} / 1.73 \mathrm{~m}^{2}$ or the presence of proteinuria) at baseline were included as study subjects in the analysis (Supplementary Fig. 1). This study was carried out in accordance with the Declaration of Helsinki. The data were anonymized and processed so that individuals could not be identified and individual privacy was maintained; thus, informed consent was waived according to the Next Generation Healthcare Infrastructure Law in Japan ${ }^{23}$. This study was approved by institutional review board [Fukuoka University Clinical Research \& Ethics Centre; FU-CREC (2017M010)]. FU-CREC also confirmed waiver of informed consent.
\end{abstract}

Definition of independent variables. At each health check, anthropometric measurements, questionnaires, and blood and urine samples were collected. We used these variables from the start of the follow-up. Blood pressure (BP) was measured in the right upper arm using mercury, automated, or aneroid sphygmomanometers with appropriately-sized cuffs after at least $5 \mathrm{~min}$ of rest in a sitting position, by trained staff. BP was measured twice and the mean of the two values was calculated. PP was calculated by subtracting the DBP from SBP. We also divided the PP into quartiles (first quartile of PP (Q1): $\leq 48, \mathrm{Q} 2: 49-56, \mathrm{Q} 3: 57-65, \mathrm{Q} 4: \geq 66$ ). The targeted $\mathrm{BP}$ was the recommended BP level $(<130 / 80 \mathrm{mmHg}$ for those with diabetes or proteinuria and $<140 / 90 \mathrm{mmHg}$ for others) according to the Japanese guidelines ${ }^{24}$. Plasma glucose levels were determined by an enzymatic method and the presence of diabetes was defined by a fasting glucose level $\geq 126 \mathrm{mg} / \mathrm{dL}$, non-fasting glucose level $\geq 200 \mathrm{mg} / \mathrm{dL}, \mathrm{HbA} 1 \mathrm{c}$ (NGSP) $\geq 6.5 \%{ }^{25}$, or use of glucose-lowering therapies. The Japanese Diabetes Society (JDS) used the JDS HbA1c instead of NGSP until the fiscal year 2012. We converted the JDS HbA1c into NGSP using the following formula: NGSP $(\%)=1.02 \times$ JDS $(\%)+0.25 \%$. Serum low-density lipoprotein (LDL) cholesterol, high-density lipoprotein (HDL) cholesterol, and triglyceride levels were determined enzymatically. Dyslipidemia was defined by LDL cholesterol $\geq 140 \mathrm{mg} / \mathrm{dL}$, HDL cholesterol $<40 \mathrm{mg} / \mathrm{dL}$, triglycerides $\geq 150 \mathrm{mg} /$ $\mathrm{dL}^{26}$, or the use of lipid-lowering medications. Serum uric acid was determined using an enzymatic method and hyperuricemia was defined by uric acid $\geq 7 \mathrm{mg} / \mathrm{dL}^{27}$. Information regarding smoking habits was obtained using a standard questionnaire. Height and body mass were measured with the participant wearing light clothes without shoes, and the body mass index (BMI, $\mathrm{kg} / \mathrm{m}^{2}$ ) was calculated. Obesity was defined as BMI $\geq 25 \mathrm{~kg} / \mathrm{m}^{228}$. Current smokers were defined as participants who had smoked 100 cigarettes or more or who had smoked continuously for more than 6 months at the baseline examination. We classified drinking as never/chance drinker and habitual drinker using a questionnaire. Serum creatinine levels were determined by an enzymatic method and eGFR was estimated using the formula of the Japanese Society of Nephrology, as follows: eGFR ( $\mathrm{mL} / \mathrm{min} / 1.73$ $\left.\mathrm{m}^{2}\right)=194 \times$ serum creatinine ${ }^{-1.094} \times$ age $^{-0.287}(\times 0.739 \text {; if female })^{29}$. Urinary protein was examined using the dipstick method and a $1+$ or higher score was regarded as indicating the presence of proteinuria ${ }^{14}$.

Definition of outcome variables. The primary outcome was progression of CKD among those with $\mathrm{CKD}$ at baseline. Progression of CKD was defined as exacerbation in the eGFR category or urinary protein category from baseline categories according to the Kidney Disease: Improving Global Outcome (KDIGO) CKD guidelines ${ }^{1}$, which was confirmed at the final follow-up examination, among participants who had CKD at baseline. eGFR categories were defined as G3a: eGFR 45-59, G3b: 30-44, G4: 15-29, and G5: $<15 \mathrm{~mL} / \mathrm{min} / 1.73 \mathrm{~m}^{2}$. Urinary protein was evaluated by urine dipstick and categorized as A1: $(-)$ or $( \pm), \mathrm{A} 2:(1+)$, and A3: $(2+)$ or more $\mathrm{e}^{5,30}$. Progression of eGFR decline was exacerbation in the eGFR category from the baseline category (Fig. 1). 
Similarly, deterioration of urinary protein was defined as exacerbation in a protein category from the baseline categories (Fig. 1). We analyzed the progression of eGFR decline or proteinuria separately for further analyses.

Statistical analysis. Baseline characteristics were compared across PP quartile categories using analysis of variance or the Kruskal-Wallis test for continuous variables and Pearson's chi-square test for categorical variables. Follow-up was continued until the first episode of outcome, the end of the study period, or censoring. Incidence was calculated using the person-year approach. The influences of PP (per $10 \mathrm{mmHg}$ ) or PP quartile on the progression of CKD were estimated using Cox's proportional hazards models. Covariates used in the analyses were sex, age, diabetes mellitus, dyslipidemia, hyperuricemia, obesity, smoking status (current or not current), drinking alcohol (habitual or never/chance), hemoglobin, and baseline eGFR and proteinuria (presence or absence). We then investigated the association between PP quartile and progression of CKD by stratifying SBP (SBP $<140 \mathrm{mmHg}, \mathrm{SBP} \geq 140 \mathrm{mmHg}$ ) and DBP (DBP < $90 \mathrm{mmHg}, \mathrm{SBP} \geq 90 \mathrm{mmHg})$. Next, we evaluated the association between PP and secondary outcomes including progression of eGFR decline and proteinuria separately. We compared the effect of PP quartile on the progression of CKD between subgroups (sex, age, diabetes mellitus, dyslipidemia, hyperuricemia, obesity, smoking status (current or not current), drinking alcohol (habitual or never/chance), hemoglobin, and baseline eGFR and proteinuria) by adding interaction terms to the models. We used the median age, hemoglobin, and baseline eGFR, and binary values of urinary protein (absent or present), for the subgroup analysis. Finally, we compared the discrimination of risk prediction between model 1 ( $<$ targeted BP or $\geq$ targeted $\mathrm{BP})$, model 2 ( $\mathrm{PP} \geq 66 \mathrm{mmHg})$, and model 3 (combination of PP $(<66$ or $\geq 66 \mathrm{mmHg}$ and/or targeted BP) using Harrell's C-index ${ }^{31}$. We used a cut-off point of PP $66 \mathrm{mmHg}$ in model 3 because CKD progression was significantly increased at the point of $\mathrm{Q} 4$ ( $\mathrm{PP} \geq 66 \mathrm{mmHg}$ ), which was similar to the cut-off of a previous study $(\mathrm{PP}>67 \mathrm{mmHg})^{21}$. Because there was high multicollinearity between continuous PP and continuous SBP $(r=0.801)$, we did not enter PP and SBP into one equation simultaneously. We found that $12.0 \%$ of the data on drinking, $0.10 \%$ on smoking status, $0.19 \%$ on BP-lowering medication, $0.38 \%$ on uric acid, and $0.76 \%$ on hemoglobin were missing. Therefore, we conducted a complete-case analysis in the primary analysis, followed by a sensitivity analysis using multiple imputation. Twenty datasets were created for multiple imputation. We regarded missing patterns as arbitrarily missing and used the Multiple Imputation by Chained Equation. STATA release 16 (STATA Corp, College Station, TX) was used for statistical analyses. All reported $P$-values were two-tailed, and the level of significance was set at $P<0.05$.

Ethics approval. This study was approved by the Fukuoka University Clinical Research \& Ethics Centre (2017M010). The members of ethics committee that involved in the study were as follow; Fumihito Hirai, Shinichiro Yasunaga, Teruaki Izaki, Ryoko Sakuma, Satoshi Imaizumi, Kohichiro Kawashima, Toshiyasu Ikuta, Maho Oishi and Yuri Kusunose.

\section{Results}

Baseline characteristics. Baseline characteristics of the subjects are shown in Table 1. Of 1042 people who were eligible for this study, the number of people in Q1, Q2, Q3, and Q4 were 278 (26.7\%), 260 (25.0\%), 245 (23.5\%), and 259 (24.9\%), respectively. Mean age, SBP, FBS, the percentage of those taking BP-lowering medications, and those with diabetes and dyslipidemia were elevated in accordance with the elevation of PP quartile. In contrast, hemoglobin and eGFR tended to decrease with a larger PP. Regarding eGFR and proteinuria, those with a more advanced condition were more likely to be in the larger quartile of PP. There was no significant relationship between sex, DBP, TG, HDL-C, LDL-C, uric acid, hyperuricemia, BMI, obesity, smoking and drinking, and PP category.

Incidences and hazard ratios associated with pulse pressure and PP quartile in the progression of chronic kidney disease. Incidences of the progression of chronic kidney disease are shown in Table 2. During a mean follow-up of 4.66 years ( 4855 person-years), there were 241 cases of CKD progression (incident rate: 49.8 per 1000 person-years). There was a significant increase in CKD progression per $10 \mathrm{mmHg}$ of PP elevation, even when adjusted for confounding factors (adjusted HR $1.17(1.06-1.29) \mathrm{p}<0.001)$. For each PP category, the incidence rates (per 1000 person-year) of Q1, Q2, Q3, and Q4 were 31.9, 40.5, 47.2, and 90.1, respectively. The crude HR of CKD progression increased linearly in accordance with larger PP [Q2: 1.27 (0.85-1.89), Q3: 1.47 (0.99-2.18), Q4: $2.72(1.89-3.91) \mathrm{p}<0.001$ for trend]. Similar linear results were obtained even after controlling for age, sex, BP-lowering medication, diabetes, dyslipidemia, hyperuricemia, obesity, smoking, drinking, hemoglobin, baseline eGFR, and proteinuria [Q2: 1.14 (0.74-1.76), Q3: 1.35 (0.88-2.06), Q4: 1.87 (1.23-2.83) $\mathrm{p}=0.003$ for trend]. There was no significant interaction between SBP, DBP, and PP for the progression of CKD $(\mathrm{p}=0.976$ for interaction between SBP $(<140 \mathrm{mmHg}$ or $\geq 140 \mathrm{mmHg})$ and $\mathrm{PP} ; \mathrm{p}=0.651$ for interaction between DBP (<90 mmHg or $\geq 90 \mathrm{mmHg}$ ) and PP) (Fig. 2).

Incidences and hazard ratios associated with pulse pressure quartile in the progression of GFR decline and proteinuria. We further separated the outcome of CKD into progression of GFR decline and proteinuria and analyzed their association with PP (Fig. 3). For the progression of GFR decline, there was a linear association with continuous PP and quartile of PP even after adjustment [continuous PP (per $10 \mathrm{mmHg}$ ); 1.17 (1.04-1.31) p =0.007; quartile of PP: Q2: 1.11 (0.67-1.83), Q3: $1.30(0.80-2.13), Q 4: 1.83(1.14-2.94) \mathrm{p}=0.010$ for trend]. For proteinuria, there was a linear tendency between continuous $\mathrm{PP}$ and progression of proteinuria although statistical significance was not achieved [continuous PP (per $10 \mathrm{mmHg}$ ); $1.17(0.98-1.40) \mathrm{p}=0.084$ ], and similar results were obtained even after adjustment [quartile of PP; Q2: 1.01 (0.47-2.14), Q3: 1.43 (0.702.91), Q4: $1.84(0.90-3.73) \mathrm{p}=0.059$ for trend] (Fig. 3). 


\begin{tabular}{|c|c|c|c|c|c|c|}
\hline & Q1 ( $\leq 48)$ & Q2 (49-56) & Q3 (57-65) & Q4 (66 $\leq)$ & \multirow[b]{2}{*}{ Total } & \multirow[b]{2}{*}{ p-value } \\
\hline & $N=278$ & $\mathrm{~N}=\mathbf{2 6 0}$ & $\mathrm{N}=245$ & $\mathrm{~N}=259$ & & \\
\hline $\begin{array}{l}\text { Age (years), } \\
\text { mean } \pm S D\end{array}$ & $62.2(9.0)$ & $64.1(6.5)$ & $64.9(5.8)$ & $67.0(5.4)$ & $64.5(7.1)$ & $<0.001$ \\
\hline Sex, men (\%) & $160(57.6)$ & $122(46.9)$ & $123(50.2)$ & $133(51.4)$ & $538(51.6)$ & 0.093 \\
\hline $\begin{array}{l}\text { Systolic BP } \\
(\mathrm{mmHg}) \\
\text { mean } \pm \text { SD }\end{array}$ & $117.5(12.2)$ & $128.4(10.4)$ & $135.9(11.7)$ & $152.2(15.8)$ & $133.2(18.0)$ & $<0.001$ \\
\hline \begin{tabular}{|l|} 
iastolic BP \\
$(\mathrm{mmHg})$, \\
mean $\pm S D$ \\
\end{tabular} & $75.7(11.0)$ & $75.9(10.1)$ & $75.3(11.4)$ & $76.5(11.8)$ & $75.9(11.1)$ & 0.620 \\
\hline $\begin{array}{l}\text { BP-lowering medi- } \\
\text { cation (\%) }\end{array}$ & $105(37.8 \%)$ & $113(43.6 \%)$ & $118(48.4 \%)$ & $147(56.8 \%)$ & $483(46.4 \%)$ & $<0.001$ \\
\hline $\begin{array}{l}\text { Fast blood sugar } \\
(\mathrm{mg} / \mathrm{dL}), \text { mean } \pm \mathrm{SD}\end{array}$ & $96.5(20.1)$ & $99.2(24.4)$ & $103.1(30.1)$ & $104.4(26.8)$ & $100.6(25.5)$ & 0.010 \\
\hline $\begin{array}{l}\text { HbAlc (\%), } \\
\text { mean } \pm \text { SD }\end{array}$ & $5.6(0.9)$ & $5.6(0.8)$ & $5.8(1.0)$ & $5.8(1.0)$ & $5.7(0.9)$ & 0.013 \\
\hline Diabetes (\%) & $30(10.8 \%)$ & $37(14.2 \%)$ & $53(21.6 \%)$ & $67(25.9 \%)$ & $187(17.9 \%)$ & $<0.001$ \\
\hline $\begin{array}{l}\text { TG (mg/dL), } \\
\text { median (Q25, Q75) }\end{array}$ & $103.0(74.0-147.0)$ & $112.5(79.0-154.0)$ & $115.0(79.0-161.0)$ & $114.0(83.0-163.0)$ & $111.0(78.0-155.0)$ & 0.057 \\
\hline $\begin{array}{l}\text { HDL-C (mg/dL), } \\
\text { median (Q25, Q75) }\end{array}$ & $59.0(47.0-74.0)$ & $57.0(47.0-70.5)$ & $57.0(48.0-70.0)$ & $56.0(46.0-67.0)$ & $57.0(47.0-70.0)$ & 0.180 \\
\hline $\begin{array}{l}\text { LDL-C (mg/dL), } \\
\text { median (Q25, Q75) }\end{array}$ & $121.0(103.0-141.0)$ & $122.0(99.5-143.0)$ & $117.0(100.0-146.0)$ & $117.0(98.0-141.0)$ & $120.0(100.0-142.0)$ & 0.630 \\
\hline Dyslipidemia (\%) & $142(51.1 \%)$ & $161(61.9 \%)$ & $136(55.5 \%)$ & $156(60.2 \%)$ & $595(57.1 \%)$ & 0.049 \\
\hline $\begin{array}{l}\text { Uric acid (mg/dL), } \\
\text { median (Q25, Q75) }\end{array}$ & $5.6(4.6-6.8)$ & $5.6(4.8-6.5)$ & $5.6(4.7-6.7)$ & $5.6(4.8-6.7)$ & $5.6(4.7-6.7)$ & 0.810 \\
\hline Hyperuricemia (\%) & $55(19.9 \%)$ & $43(16.5 \%)$ & $56(23.1 \%)$ & $54(20.8 \%)$ & $208(20.0 \%)$ & 0.310 \\
\hline $\begin{array}{l}\mathrm{BMI}\left(\mathrm{kg} / \mathrm{m}^{2}\right) \\
\text { mean } \pm \mathrm{SD}\end{array}$ & $24.1(3.8)$ & $24.3(3.4)$ & $24.3(3.5)$ & $24.9(3.8)$ & $24.4(3.6)$ & 0.062 \\
\hline Obesity (\%) & $110(39.6 \%)$ & $105(40.4 \%)$ & $99(40.4 \%)$ & $121(46.7 \%)$ & $435(41.7 \%)$ & 0.310 \\
\hline \multicolumn{7}{|l|}{ Smoking, n (\%) } \\
\hline Current smoker & $49(17.6 \%)$ & $34(13.1 \%)$ & $39(16.0 \%)$ & $34(13.1 \%)$ & $156(15.0 \%)$ & 0.370 \\
\hline \multicolumn{7}{|l|}{ Alcohol, n (\%) } \\
\hline $\begin{array}{l}\text { Never/chance } \\
\text { drinker (\%) }\end{array}$ & $198(79.2 \%)$ & $189(79.7 \%)$ & $162(76.8 \%)$ & $171(78.1 \%)$ & $720(78.5 \%)$ & 0.880 \\
\hline $\begin{array}{l}\text { Habitual drinker } \\
(\%)\end{array}$ & $52(20.8 \%)$ & $48(20.3 \%)$ & $49(23.2 \%)$ & $48(21.9 \%)$ & $197(21.5 \%)$ & \\
\hline $\begin{array}{l}\text { Hemoglobin, } \\
\text { mean } \pm \text { SD }\end{array}$ & $14.1(1.6)$ & $14.0(1.4)$ & $13.8(1.4)$ & $13.6(1.6)$ & $13.9(1.5)$ & $<0.001$ \\
\hline eGFR, mean \pm SD & $59.4(15.8)$ & $57.0(12.4)$ & $57.1(13.3)$ & $56.2(16.2)$ & $57.5(14.6)$ & 0.065 \\
\hline \multicolumn{7}{|l|}{ KDIGO classification } \\
\hline \multicolumn{7}{|l|}{ eGFR categories } \\
\hline G1 $(\mathrm{eGFR} \geq 90)$ & $67(24.1 \%)$ & $51(19.6 \%)$ & $46(18.8 \%)$ & $61(23.6 \%)$ & $225(21.6 \%)$ & 0.051 \\
\hline G2 (eGFR 60-89) & $195(70.1 \%)$ & $187(71.9 \%)$ & $176(71.8 \%)$ & $164(63.3 \%)$ & $722(69.3 \%)$ & \\
\hline G3 (eGFR 30-59) & $13(4.7 \%)$ & $20(7.7 \%)$ & $19(7.8 \%)$ & $22(8.5 \%)$ & $74(7.1 \%)$ & \\
\hline G4 (eGFR 15-29) & $1(0.4 \%)$ & $1(0.4 \%)$ & $3(1.2 \%)$ & $6(2.3 \%)$ & $11(1.1 \%)$ & \\
\hline G5 $($ eGFR $<15)$ & $2(0.7 \%)$ & $1(0.4 \%)$ & $1(0.4 \%)$ & $6(2.3 \%)$ & $10(1.0 \%)$ & \\
\hline \multicolumn{7}{|l|}{ Proteinuria categories } \\
\hline $\begin{array}{l}\text { A1 (proteinuria } \\
(-) /( \pm))\end{array}$ & $190(68.3 \%)$ & $191(73.5 \%)$ & $169(69.0 \%)$ & $157(60.6 \%)$ & $707(67.9 \%)$ & 0.011 \\
\hline $\begin{array}{l}\text { A2 (proteinuria } \\
(+))\end{array}$ & $64(23.0 \%)$ & $49(18.8 \%)$ & $52(21.2 \%)$ & $59(22.8 \%)$ & $224(21.5 \%)$ & \\
\hline $\begin{array}{l}\text { A3 (proteinuria } \\
(2+) \sim)\end{array}$ & $24(8.6 \%)$ & $20(7.7 \%)$ & $24(9.8 \%)$ & $43(16.6 \%)$ & $111(10.7 \%)$ & \\
\hline
\end{tabular}

Table 1. Baseline characteristics of study participants by pulse pressure quartile. eGFR estimated glomerular filtration rate, KDIGO Kidney Disease: Improving Global Outcome, $B M I$ body mass index, $T G$ triglyceride, $H D L-c$ high-density lipoprotein cholesterol, $L D L-C$ low-density lipoprotein cholesterol, $B P$ blood pressure, $S D$ standard deviation.

Results of multiple imputation. Sensitivity analysis using the multiple imputation of missing values demonstrated comparable findings: Q2: 1.22 (0.82-1.84), Q3: 1.25 (0.83-1.88), Q4: 1.86 (1.27-2.74) for progres- 


\begin{tabular}{|c|c|c|c|c|c|}
\hline & Incident rate (per 1000 person-years) & Crude HR (95\% CI) & p-value & Adjusted HR & p-value \\
\hline \multicolumn{6}{|l|}{ Progression of CKD } \\
\hline $\mathrm{PP}($ per $10 \mathrm{mmHg})$ & - & $1.30(1.20-1.42)$ & $<0.001$ & $1.17(1.06-1.29)$ & 0.002 \\
\hline \multicolumn{6}{|l|}{ PP quartile } \\
\hline $\mathrm{Q} 1(\leq 48)$ & $31.9(44 / 1379)$ & 1.00 (reference) & \multirow{4}{*}{$<0.001^{*}$} & 1.00 (reference) & \multirow{4}{*}{$0.003^{*}$} \\
\hline Q2 (49-56) & $40.5(54 / 1333)$ & $1.14(0.85-1.89)$ & & $1.14(0.74-1.76)$ & \\
\hline Q3 (57-65) & $47.2(55 / 1166)$ & $1.47(0.99-2.18)$ & & $1.35(0.88-2.06)$ & \\
\hline Q4 ( $\geq 66)$ & $90.1(88 / 977)$ & $2.72(1.89-3.91)$ & & $1.87(1.23-2.83)$ & \\
\hline
\end{tabular}

Table 2. Incidences and hazard ratios associated with pulse pressure quartile for progression of chronic kidney disease. PP Pulse Pressure, CKD Chronic kidney disease, HR Hazard ratio, CI confidence interval. Adjusted HRs were obtained, controlling for age, sex, BP-lowering medication, diabetes, dyslipidemia, hyperuricemia, obesity, smoking, drinking, and baseline eGFR and proteinuria. ${ }^{\star} \mathrm{p}$ for trend.

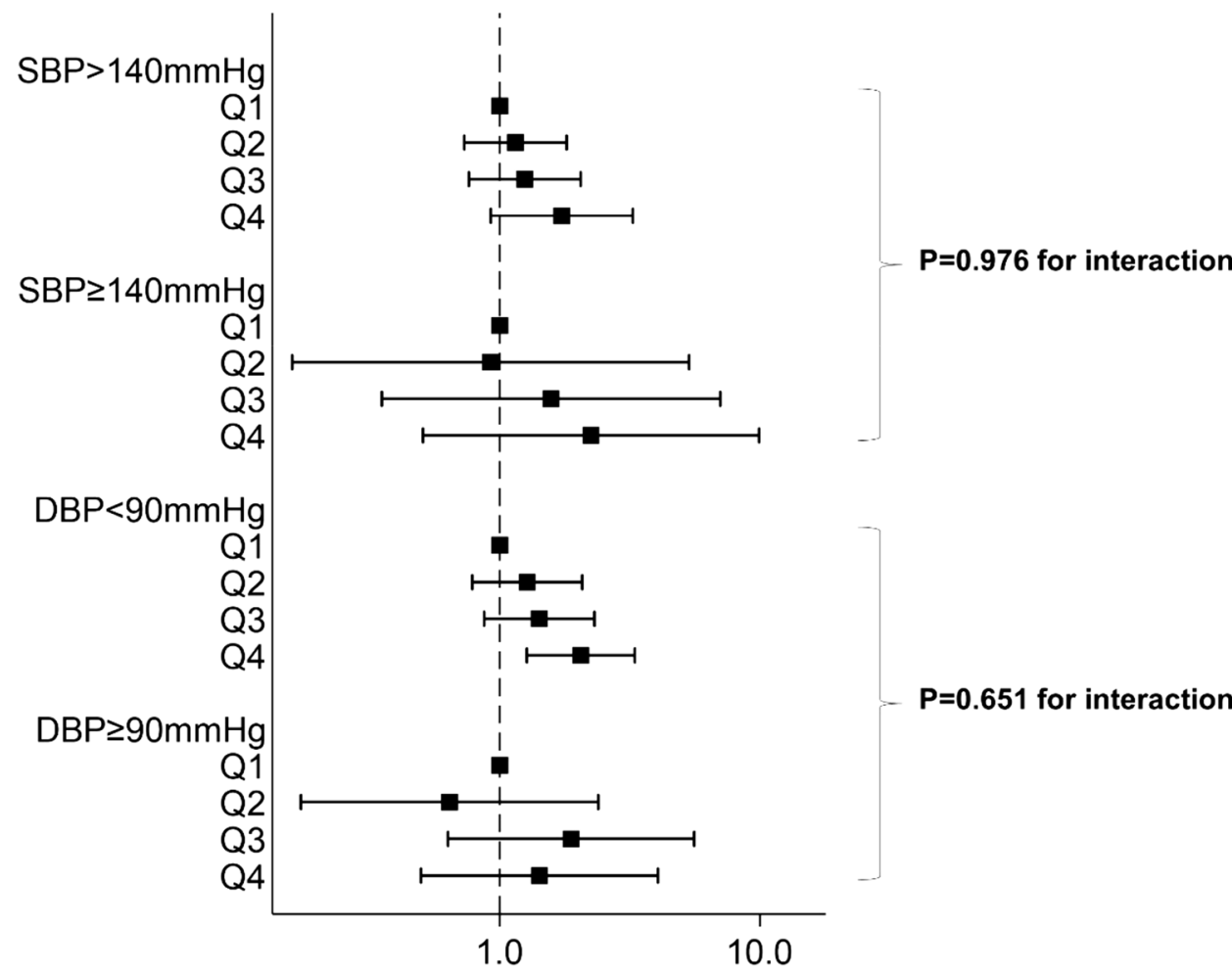

Adjusted hazard ratio (95\% confidence interaval)

Figure 2. Stratified analyses of blood pressure: relationship between pulse pressure quartile and progression of CKD. Analyses were stratified by SBP $(<140, \geq 140)$ and DBP $(<90, \geq 90)$. Adjusted hazard ratios and $95 \%$ confidence intervals associated with pulse pressure quartiles for the progression of CKD were obtained, controlling for sex, age, diabetes mellitus, dyslipidemia, hyperuricemia, obesity, current smoking and drinking alcohol, and baseline eGFR and proteinuria. $\mathrm{P}$ for interaction was obtained by adding interaction terms to the models. Boxes and vertical lines represent hazard ratios and $95 \%$ confident intervals, respectively.

sion of CKD; Q2: 1.14 (0.72-1.82), Q3: 1.17 (0.74-1.85), Q4: 1.71 (1.10-2.63) for progression of GFR decline; Q2: 1.05 (0.52-2.12), Q3: 1.39 (0.71-2.72), Q4: 1.84 (0.95-3.57) for progression of proteinuria (Supplementary Table 1).

Interaction between pulse pressure quartile and each subgroup in the progression of CKD. Figure 4 shows the effect of PP quartile on the progression of CKD by subgroup. There were significant interactions between PP and baseline $\operatorname{CKD}(<57, \geq 57)(\mathrm{p}=0.022)$ or baseline urinary protein (absent or present) $(p=0.048)$. Statistically significant interactions were not detected for other subgroups $(p>0.1)$. 


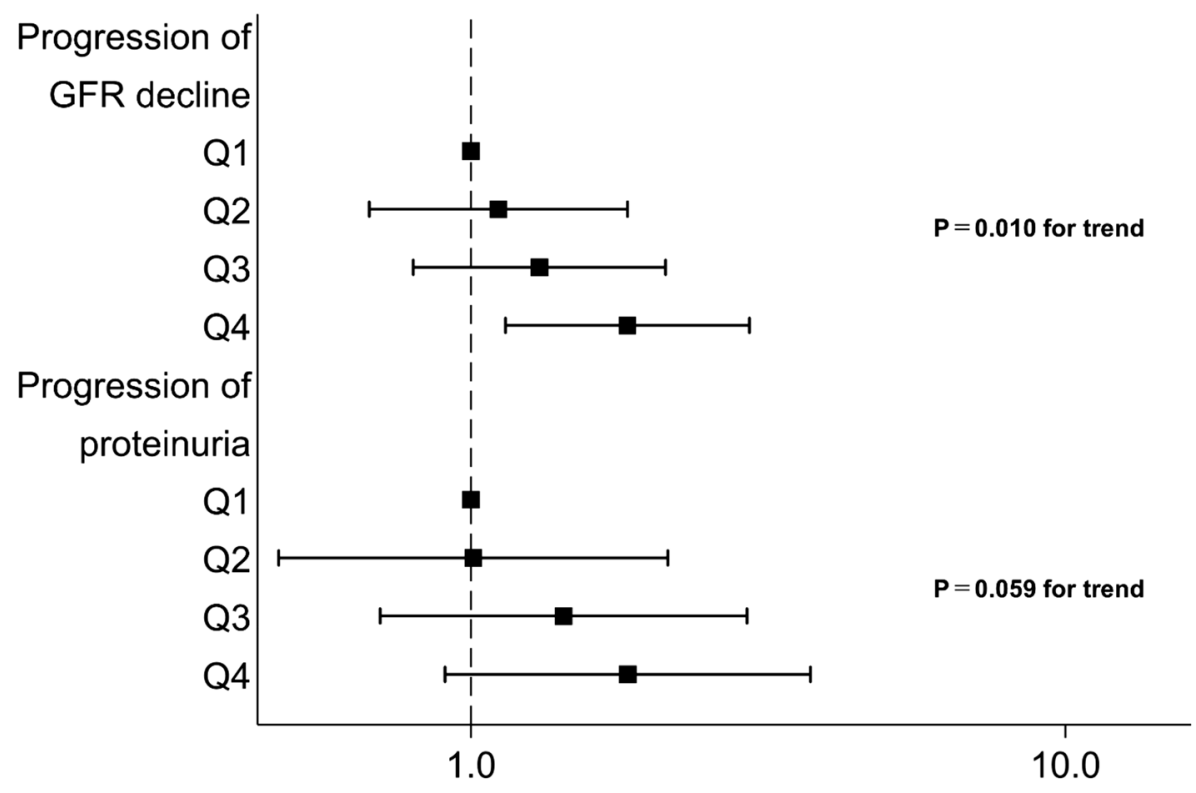

Adjusted hazard ratio (95\% confidence interaval)

Figure 3. Effects of pulse pressure quartile on eGFR decline or progression of proteinuria. Adjusted hazard ratios and 95\% confidence intervals associated with pulse pressure quartile for progression of eGFR decline or proteinuria were obtained, controlling for sex, age, diabetes mellitus, dyslipidemia, hyperuricemia, obesity, current smoking and drinking alcohol, and baseline eGFR and proteinuria. Boxes and vertical lines represent hazard ratios and $95 \%$ confident intervals, respectively.

Comparison of the discrimination of prediction between $\mathrm{PP}$, targeted $\mathrm{BP}$, and the combination of PP and targeted BP. Table 3 shows a comparison of the discrimination of prediction between model 1 (targeted $\mathrm{BP}$ ), model 2 ( $\mathrm{PP} \geq 66 \mathrm{mmHg}$ ) and model 3 (combination of $\mathrm{PP} \geq 66 \mathrm{mmHg}$ and/or targeted BP). Harrel's C-index for models 1, 2, and 3 was 0.7327 95\% CI (0.6986-0.7668), $0.735695 \%$ CI (0.7010-0.7702), and $0.733095 \% \mathrm{CI}(0.6988-0.7672)$, respectively. Dichotomous PP or adding dichotomous PP to targeted BP did not significantly improve the conventional targeted BP (differences between model 2 and model 1 , and model 3 and model 1 were $0.0029(-0.0076-0.0135) \mathrm{p}=0.588$ and $0.0003(-0.0043-0.0049) \mathrm{p}=0.854$, respectively).

\section{Discussion}

In this study, we found a significant association between PP and progression of CKD. When PP was separated into quartiles, there was a significant increase in CKD progression at $\mathrm{PP} \geq 66 \mathrm{mmHg}$, and CKD progression was also linearly increased in accordance with PP increase. This finding did not change significantly irrespective of baseline SBP or DBP. PP was still a potential predictive marker, especially for GFR decline, when we separated the outcome to progression of GFR decline and proteinuria. Although the discrimination of prediction for the progression of CKD was obtained with conventional targeted BP, there was no additional benefit of PP, or adding $\mathrm{PP}$ to the conventional targeted BP category.

PP is defined as the difference between the SBP and DBP, and is also determined by stroke volume and the compliance of large arteries ${ }^{32}$. A high PP has been well-established among elderly populations ${ }^{33}$ and those with diabetes $^{34}, \mathrm{CKD}^{35}$, or ESRD ${ }^{36}$, and it also indicated large artery stiffness ${ }^{20,37}$ as a result of arteriosclerosis ${ }^{38}$. Previous studies reported PP was a potential marker for atherosclerotic diseases such as cardiovascular disease ${ }^{16-20}$, heart failure ${ }^{16,21}$, and stroke ${ }^{16}$. The present study revealed that PP is a potential risk marker for the progression of CKD. Individuals with high PP might already have stiff vessels, which could lead to microvascular kidney disease, or their BP might be uncontrolled because of low DBP. Furthermore, the kidney is a highly perfused organ with low resistance $\mathrm{e}^{39,40}$. As aortic stiffness increases, indicated by increased PP, the kidney experiences greater pressure fluctuation and wave reflection, which leads to excessive pressure and flow pulsatility into the microvascular bed of the kidneys. Kidney arteries are subjected to high pulsate circumferential stress and high longitudinal shear stress $^{41}$, which might cause microvascular ischemia and renal tissue damage ${ }^{39}$. Thus, increased PP, which is an index of arterial stiffness, might lead to glomerular hypertrophy, hyperfiltration, segmental glomerular sclerosis, and eventually, nephrosclerosis and fibrosis ${ }^{39}$.

The Framingham study reported PP was a stronger marker for coronary heart disease than $\mathrm{BP}^{20,39}$. However, a meta-analysis by the Japan Arteriosclerosis Longitudinal Study-Existing Cohort Combine Group reported that PP was a less important predictor for cardiovascular disease $\mathrm{e}^{42}$. Our study revealed that although PP was still a potential predictive marker for CKD progression, there was no significant improvement in prediction when using 

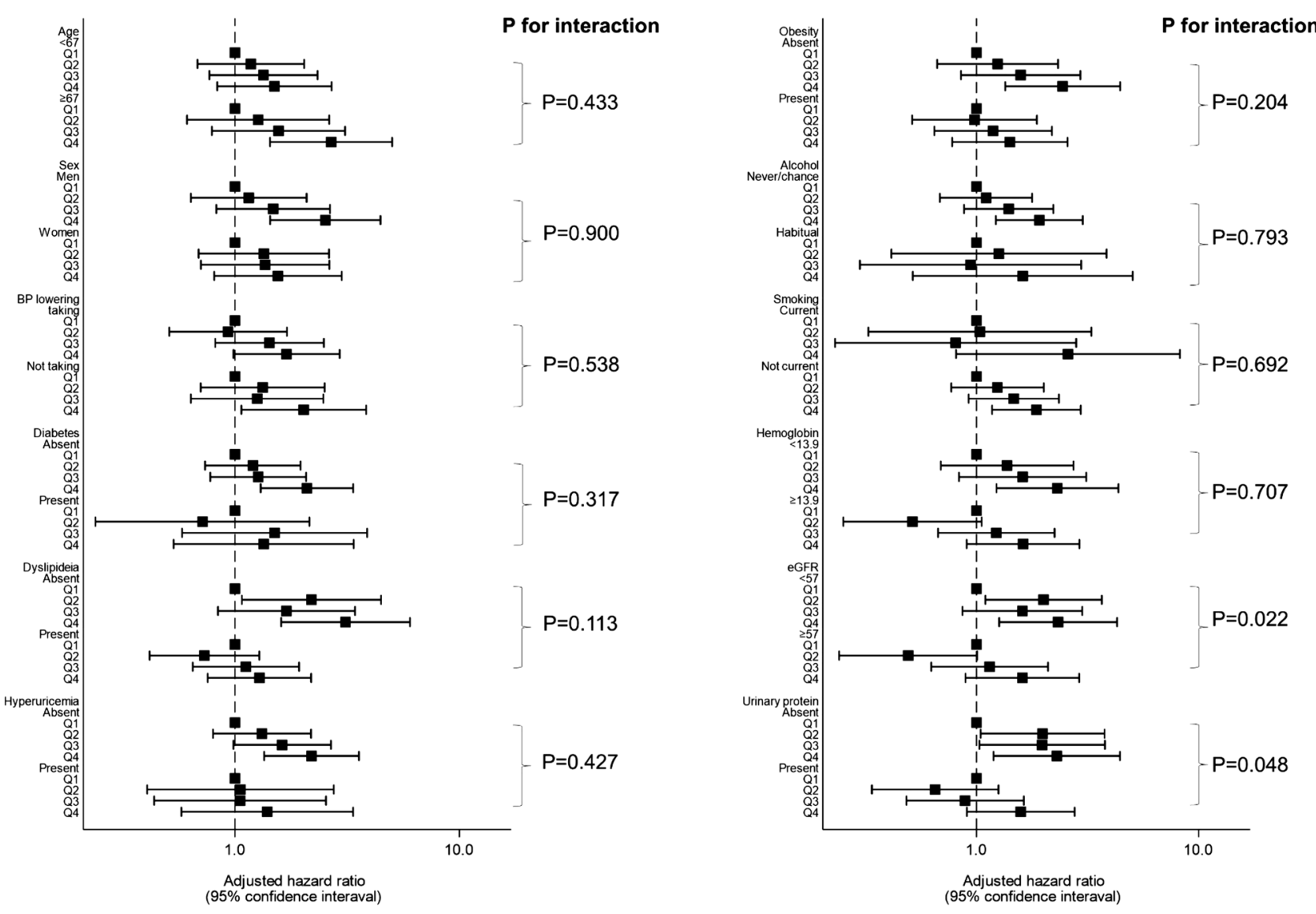

Figure 4. Subgroup analyses: interaction between pulse pressure and each covariate for the progression of CKD. Adjusted hazard ratios and 95\% confidence intervals associated with pulse pressure quartile for the progression of CKD were obtained by adding interaction terms to Cox's proportional hazards models, controlling for sex, age, diabetes mellitus, dyslipidemia, hyperuricemia, obesity, current smoking and drinking alcohol, hemoglobin, and baseline eGFR and proteinuria. $P$ for interaction was obtained by adding interaction terms to the models. Boxes and vertical lines represent hazard ratios and $95 \%$ confident intervals, respectively.

\begin{tabular}{|l|l|l|c|}
\hline & Harrel's C-index & Difference from model 1 & p-value \\
\hline Model 1 & $0.7327(0.6986-0.7668)$ & - & - \\
\hline Model 2 & $0.7356(0.7010-0.7702)$ & $0.0029(-0.0076-0.0135)$ & 0.588 \\
\hline Model 3 & $0.7330(0.6988-0.7672)$ & $0.0003(-0.0043-0.0049)$ & 0.854 \\
\hline
\end{tabular}

Table 3. Comparison of the discrimination of prediction for the progression of CKD between targeted BP, PP, and the combination of PP and targeted BP. Model 1: conventional targeted BP $(<130 / 80 \mathrm{mmHg}$ for those with diabetes or proteinuria and $<140 / 90 \mathrm{mmHg})$; model 2: $\mathrm{PP} \geq 66 \mathrm{mmHg}$; model 3: combination of PP $(<66$ or $\geq 66$ ) and/or targeted BP. Other variables for adjustment were age, sex, BP-lowering medication, diabetes, dyslipidemia, hyperuricemia, obesity, smoking, drinking, and baseline eGFR and proteinuria.

PP or adding PP to conventional targeted BP. These differences in study findings might be related to the difference in outcomes, ethnicity, or study design. Another possibility might be differences in the statistical models used-our study did not enter PP and SBP simultaneously into one equation because of high multicollinearity. As an alternative, we used Harrel's C-index to compare the discrimination of prediction for different models in contrast to previous studies that used the likelihood ratio test ${ }^{20,39}$.

The results of our subgroup analyses showed PP was affected by the extent of eGFR or proteinuria. Interestingly, PP seemed to be more related to CKD progression for those with lower eGFR or without proteinuria, suggesting proteinuria is a greater risk factor for CKD progression than PP. Another hypothesis is that two pathophysiologic mechanisms-ischemia and loss of autoregulation-are involved in hypertension-related renal disease $^{43,44}$. Proteinuria is strongly related to hyper-infiltration caused by the loss of autoregulation ${ }^{43}$. However, increased PP might be closely related to parenchymal ischemia associated with the narrowed lumen of affected vessels rather than the loss of autoregulation ${ }^{45}$, which was closely related to GFR decline ${ }^{33}$. However, further studies to confirm this are needed. 
Although this was a large-scale observational study of the general Japanese population, there were some limitations. First, because the study design was observational, we could not clarify a causal relationship. Second, the findings of the present analysis may be affected by selection bias because people with healthy lifestyles are more likely to attend health check-ups conducted by the local government than those with unhealthy lifestyles. Third, different sphygmomanometers were used, although BP was measured by trained staff according to standardized guidelines. Fourth, we did not determine competing risks, for example, death, because of data limitations. Finally, the accuracy of the dipstick method for the diagnosis of proteinuria is limited.

\section{Conclusion}

We found a significant association between PP and progression of CKD. PP might be a potential predictive marker for the progression of CKD.

\section{Data availability}

The datasets used in this study are not publicly available due to the privacy policy of the data provider. However, the data are available from the corresponding author on reasonable request.

Received: 27 August 2021; Accepted: 23 November 2021

Published online: 02 December 2021

\section{References}

1. Group KDIGO (KDIGO) CW. KDIGO 2012 clinical practice guideline for the evaluation and management of chronic kidney disease. Kidney Int. Suppl. 3(1), 4 (2013).

2. Chen, T. K., Knicely, D. H. \& Grams, M. E. Chronic kidney disease diagnosis and management: A review. JAMA J. Am. Med. Assoc. 322(13), 1294-1304 (2019).

3. Webster, A. C., Nagler, E. V., Morton, R. L. \& Masson, P. Chronic kidney disease. Lancet 389(10075), 1238-1252. https://doi.org/ 10.1016/S0140-6736(16)32064-5 (2017).

4. Matsushita, K. et al. Estimated glomerular filtration rate and albuminuria for prediction of cardiovascular outcomes: A collaborative meta-analysis of individual participant data. Lancet Diabetes Endocrinol. 3(7), 514-525 (2015).

5. Van Der Velde, M. et al. Lower estimated glomerular filtration rate and higher albuminuria are associated with all-cause and cardiovascular mortality: A collaborative meta-analysis of high-risk population cohorts. Kidney Int. 79(12), 1341-1352. https:// doi.org/10.1038/ki.2010.536 (2011).

6. Jha, V., Wang, A. Y. M. \& Wang, H. The impact of CKD identification in large countries: The burden of illness. Nephrol. Dial. Transplant. 27(SUPPL 3), 32-38 (2012).

7. Higashiyama, A. et al. Effect of chronic kidney disease on individual and population medical expenditures in the Japanese population. Hypertens. Res. 32(6), 450-454 (2009).

8. Cheung, A. K. et al. Effects of intensive BP control in CKD. J. Am. Soc. Nephrol. 28(9), 2812-2823. https://doi.org/10.1681/ASN. 2017020148 (2017).

9. De Galan, B. E. et al. Lowering blood pressure reduces renal events in type 2 diabetes. J. Am. Soc. Nephrol. 20(4), 883-892 (2009).

10. Uzu, T. et al. The effects of blood pressure control levels on the renoprotection of type 2 diabetic patients without overt proteinuria. J. Am. Soc. Hypertens. 6(2), 124-131 (2012).

11. Anonymous. Intensive blood-glucose control with sulphonylureas or insulin compared with conventional treatment and risk of complications in patients with type 2 diabetes (UKPDS 33). Lancet 352(9131), 837-853 (1998).

12. Ismail-Beigi, F. et al. Effect of intensive treatment of hyperglycaemia on microvascular outcomes in type 2 diabetes: An analysis of the ACCORD randomised trial. Lancet 376(9739), 419-430. https://doi.org/10.1016/S0140-6736(10)60576-4 (2010).

13. ADVANCE Collaborative Group. Intensive blood glucose control and vascular outcomes in patients with type 2 diabetes. N. Engl. J. Med. 358(24), 2560-2572 (2008).

14. Yamagata, K. et al. Risk factors for chronic kidney disease in a community-based population: A 10-year follow-up study. Kidney Int. 71(2), 159-166. https://doi.org/10.1038/sj.ki.5002017 (2007).

15. Ishizaka, N. et al. Association between cigarette smoking and chronic kidney disease in Japanese men. Hypertens. Res. 31(3), 485-492 (2008).

16. Vaccarino, V. et al. Pulse pressure and risk of cardiovascular events in the systolic hypertension in the elderly program. Am. J. Cardiol. 88(9), 980-986 (2001).

17. Selvaraj, S. et al. Pulse pressure and risk for cardiovascular events in patients with atherothrombosis from the REACH registry. J. Am. Coll. Cardiol. 67(4), 392-403 (2016).

18. Blacher, J. et al. Pulse pressure not mean pressure determines cardiovascular risk in older hypertensive patients. Arch. Intern Med. 160(8), 1085-1089 (2000).

19. Millar, J. A., Lever, A. F. \& Burke, V. Pulse pressure as a risk factor for cardiovascular events in the MRC Mild hypertension trial. J. Hypertens. 17(8), 1065-1072 (1999).

20. Franklin, S. S., Khan, S. A., Wong, N. D., Larson, M. G. \& Levy, D. Is pulse pressure useful in predicting risk for coronary heart disease? The Framingham Heart Study. Circulation 100(4), 354-360 (1999).

21. Chae, C. U. et al. Increased pulse pressure and risk of heart failure in the elderly. J. Am. Med. Assoc. 281(7), 634-639 (1999),

22. Maeda, T. et al. Usefulness of the blood pressure classification in the new 2017 ACC/AHA hypertension guidelines for the prediction of new-onset chronic kidney disease. J. Hum. Hypertens. 33(12), 873-878. https://doi.org/10.1038/s41371-019-0198-7 (2019).

23. Yasunaga, H. Protection of personal information in real-world data in Japan. Ann. Clin. Epidemiol. 2(1), 1-2 (2020).

24. Japanese Society of Nephrology. Evidence-based clinical practice guideline for CKD 2013. Clin. Exp. Nephrol. 18(3), 346-423 (2014).

25. Araki, E. et al. Japanese clinical practice guideline for diabetes 2019. J. Diabet. Investig. 11, 1020-1076 (2020).

26. Kinoshita, M. et al. Japan Atherosclerosis Society (JAS) guidelines for prevention of atherosclerotic cardiovascular diseases 2017. J. Atheroscler. Thromb. 25(9), 846-984 (2018).

27. Yamanaka, H. Japanese guideline for the management of hyperuricemia and gout: Second edition. Nucleosid. Nucleot. Nucleic Acids 30(12), 1018-1029 (2011).

28. Matsuzawa, Y. et al. New criteria for "obesity disease" in Japan. Circ. J. 66(11), 987-992 (2002).

29. Matsuo, S. et al. Revised equations for estimated GFR from serum creatinine in Japan. Am. J. Kidney Dis. 53(6), 982-992. https:// doi.org/10.1053/j.ajkd.2008.12.034 (2009).

30. Gansevoort, R. T. et al. Lower estimated GFR and higher albuminuria are associated with adverse kidney outcomes: A collaborative meta-analysis of general and high-risk population cohorts. Kidney Int. 80(1), 93-104. https://doi.org/10.1038/ki.2010.531 (2011).

31. Newson, R. B. Comparing the predictive powers of survival models using Harrell's C or Somers' D. Stata J. 10(3), 339-358 (2010). 
32. Dart, A. M. K. B. Pulse pressure: A review of mechanisms and clinical relevance. J. Am. Coll. Cardiol. 37, 975-984 (2001).

33. Verhave, J. C. et al. Elevated pulse pressure is associated with low renal function in elderly patients with isolated systolic hypertension. Hypertension 45(4), 586-591 (2005).

34. Vaccarino, V., Holford, T. R. \& Krumholz, H. M. Pulse pressure and risk for myocardial infarction and heart failure in the elderly. J. Am. Coll. Cardiol. 36(1), 130-138. https://doi.org/10.1016/S0735-1097(00)00687-2 (2000).

35. Iseki, K. et al. Low diastolic blood pressure, hypoalbuminemia, and risk of death in a cohort of chronic hemodialysis patients. Kidney Int. 51(4), 1212-1217 (1997).

36. Klassen, P. S. et al. Association between pulse pressure and mortality in patients undergoing maintenance hemodialysis. JAMA 287(12), 1548-1555 (2002).

37. Nair, G. V. et al. Pulse pressure and coronary atherosclerosis progression in postmenopausal women. Hypertension 45(1), 53-57 (2005).

38. Arulkumaran, N. et al. Pulse pressure and progression of chronic kidney disease. J. Nephrol. 23(2), 189-193 (2010).

39. Geng, T. T., Talaei, M., Jafar, T. H., Yuan, J. M. \& Koh, W. P. Pulse pressure and the risk of end-stage renal disease among Chinese adults in Singapore: The Singapore Chinese health study. J. Am. Heart Assoc. 8, 23 (2019).

40. Briet, M., Boutouyrie, P., Laurent, S. \& London, G. M. Arterial stiffness and pulse pressure in CKD and ESRD. Kidney Int. 82(4), 388-400 (2012).

41. O’Rourke, M. F. \& Safar, M. E. Relationship between aortic stiffening and microvascular disease in brain and kidney: Cause and logic of therapy. Hypertension 46(1), 200-204 (2005).

42. Miura, K. et al. Four blood pressure indexes and the risk of stroke and myocardial infarction in Japanese men and women a metaanalysis of 16 cohort studies. Circulation 119(14), 1892-1898 (2009).

43. Hill, G. S. Hypertensive nephrosclerosis. Curr. Opin. Nephrol. Hypertens. 17(3), 266-270 (2008).

44. Meyrier, A. Nephrosclerosis: Update on a centenarian. Nephrol. Dial. Transplant. 30(11), 1833-1841 (2015).

45. Vinay Kumar, A. K. \& Abbas, J. C. A. Robbins \& Cotran Pathologic Basis of Disease 10th edn. (Elsevier, 2020).

\section{Acknowledgements}

We thank J. Ludovic Croxford, $\mathrm{PhD}$, from Edanz (https://jp.edanz.com/ac) for editing a draft of this manuscript. This work was supported by the Fukuoka University Recommendation Project (Grant Number 207106-000) and JSPS KAKENHI (Grant Numbers 18K17404, 19H03879, 20K20674, and 21K17317). HA received research grants from Daiichi Sankyo, Eisai, and Takeda, lecture fees from Bayer, Daiichi Sankyo, Fukuda Denshi, MSD, Takeda, and Teijin, and nonfinancial research support (rental equipment) from Phillips. CY was funded by the Fukuoka University Recommendation Project (Grant Number 197006-000), JSPS KAKENHI (Grant Numbers $18 \mathrm{~K} 10078$ and $21 \mathrm{~K} 10486$ ) and received other financial or non-financial interests (Endowed Course from Fukuda Denshi, Fukuda Lifetec Kyushu). TN was funded by JSPS KAKENHI (Grant Numbers 19H03879 and 20K20674).

\section{Author contributions}

T.M., S.Y., S.F., M.T., A.S., M.A., M.K., C.Y., Ka.T., Ko.T., K.I., Te.Y., To.Y., K.I., A.M., K.M., T.U., K.M., S.M., and H.A. contributed to data acquisition. T.M., S.Y., T.N., and H.A. conceived, designed, and analyzed the data. T.M. wrote the manuscript with input from all authors. All authors read the manuscript and revised it critically for important intellectual content. All authors approved the final version of the manuscript to be published. All authors agreed to be accountable for all aspects of the work in ensuring that questions related to the accuracy or integrity of any part of the work are appropriately investigated and resolved.

\section{Funding}

This work was supported by Fukuoka University Recommendation Project (Grant Number 207106-000) and JSPS KAKENHI (Grant Numbers 18K17404, 19H03879, 20K20674, and 21K17317).

\section{Competing interests}

TM was funded by JSPS KAKENHI (Grant Numbers 18K17404 and 21K17317). HA received research grants from Daiichi Sankyo, Eisai, and Takeda, lecture fees from Bayer, Daiichi Sankyo, Fukuda Denshi, MSD, Takeda, and Teijin, and nonfinancial research support (rental equipment) from Phillips. CY was funded by the Fukuoka University Recommendation Project (Grant Number 197006-000), JSPS KAKENHI (Grant Numbers 18K10078 and $21 \mathrm{~K} 10486$ ), and received other financial or non-financial interests (Endowed Course from Fukuda Denshi, Fukuda Lifetec Kyushu). TN was funded by JSPS KAKENHI (Grant Numbers 19H03879 and 20K20674). Other authors (SY, SF, MT, AS, MA, MK, KaT, KoT, KI, TeY, ToY, KI, AM, KM, TU, KM, and SM) have no competing interests to declare.

\section{Additional information}

Supplementary Information The online version contains supplementary material available at https://doi.org/ 10.1038/s41598-021-02809-8.

Correspondence and requests for materials should be addressed to T.M.

Reprints and permissions information is available at www.nature.com/reprints.

Publisher's note Springer Nature remains neutral with regard to jurisdictional claims in published maps and institutional affiliations. 
(c) (i) Open Access This article is licensed under a Creative Commons Attribution 4.0 International cc) License, which permits use, sharing, adaptation, distribution and reproduction in any medium or format, as long as you give appropriate credit to the original author(s) and the source, provide a link to the Creative Commons licence, and indicate if changes were made. The images or other third party material in this article are included in the article's Creative Commons licence, unless indicated otherwise in a credit line to the material. If material is not included in the article's Creative Commons licence and your intended use is not permitted by statutory regulation or exceeds the permitted use, you will need to obtain permission directly from the copyright holder. To view a copy of this licence, visit http://creativecommons.org/licenses/by/4.0/.

(C) The Author(s) 2021 